SESSION 6

PHYSICS OF THE NEUTRON STAR 


\title{
6.1 PHYSICS OF THE NEUTRON STAR
}

\author{
A. G. W. CAMERON \\ Belfer Graduate School of Science, Yeshiva University, New York, N.Y., U.S.A., and \\ Goddard Institute for Space Studies, NASA, New York, N.Y., U.S.A.
}

\begin{abstract}
Some characteristics of neutron star models are described. These include: the physics of the crystalline crust, the superfluid properties of the neutrons and protons, and the presence of rotational vortices in these superfluids. The possible relationship of some of these properties to the behavior of the Crab Nebula pulsar is described.
\end{abstract}

The discovery of pulsars has focused the attention of theoretical physicists upon neutron stars, with which the pulsars have been identified. Essentially every major field of theoretical physics is represented in the investigation of the neutron star (Cameron, 1970), and in most of these fields the problems posed lie at the very forefront of research, since the conditions in the neutron star are so extremely unlike those encountered in the laboratory. The Crab Nebula has long had the reputation of exhibiting a greater variety of physical phenomena than any other object in the sky, and this characteristic also seems to be present in the Crab Nebula pulsar, which exhibits a greater variety of phenomena than seen in any of the other pulsars.

In Figure 1 the pie-shaped diagram shows a section of a model of a neutron star. The star can be considered to have three major distinct regions. In the outermost region, the neutron-rich nuclei are present, which are expected to form a rigid crystalline lattice. Near the base of this crystal, neutrons become interspersed with the nuclei, and finally, just before the nuclei disappear with increasing density, some protons also become interspersed with the nuclei. The second region, lying toward the intermediate radial distances, consists of a neutron-proton-electron fluid. In the higher density regions of this part, negative mu mesons also become present. In the third and innermost part, hyperons also first put in their appearance, starting with the $\Sigma^{-}$hyperon (Langer et al., 1969; Langer and Rosen, 1970).

The determination of the precise composition of the neutron star interior is intimately related to the determination of the appropriate equation of state. The calculation of the equation of state is based upon the usual and reasonable assumption that the interior of the star is at zero temperature, since the Fermi levels of all the degenerate particles are enormously high compared to the typical thermal energies of the particles in the interior. The determination of the equation of state requires the minimization of the total energy per baryon in each range of density in the interior. Among the energies which enter into the minimization calculation are the potential energies between the baryons. These potential energies are still very imperfectly understood, since densities both high and low compared to normal nuclear density are involved in the neutron star interior, and nuclear forces themselves are still relatively poorly understood. The models of neutron stars which are presented in this paper are based upon an equation of state for the full density range of the neutron star interior calcu- 


\section{COMPOSITION AND STRUCTURE OF $0.586 M_{\odot}$ NEUTRON STAR RADIUS $=13.7 \mathrm{KM}$}

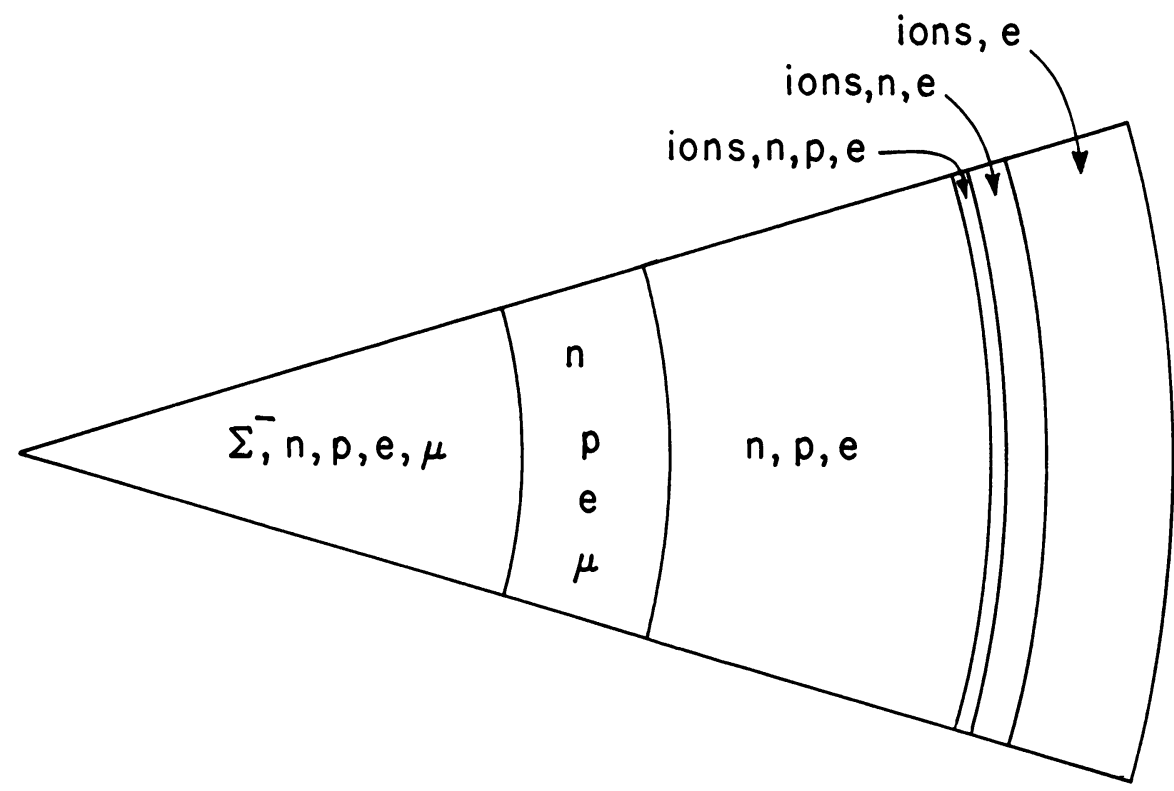

Fig. 1. Section of a neutron star model showing the constituents. The distances are to scale.

lated by Langer, Rosen, Cohen, and myself (Langer et al., 1969; Langer and Rosen, 1970). This equation of state is relatively stiff at greater than normal nuclear densities, and leads to rather high permitted masses for neutron stars. There have been other equations of state calculated in the last two years and applied to the determination of neutron star models, which differ quite markedly from our own. We are biased and prefer our own equation of state, because we know that it can reproduce the properties of ordinary nuclei rather well. Some of the other equations of state do not succeed so well in this respect.

The neutron star models which we have obtained possess the relation between central density and mass shown in Figure 2 (Cohen et al., 1970; Cohen and Cameron, 1970). In the left portion of this figure there is a peak which corresponds to white dwarf stars. Those models on the left hand side of the peak are stable, whereas those on the right of the peak are unstable against collapse. The neutron star peak is the large one on the right; the intermediate structure which appears to have an additional peak does not correspond to stable models in hydrostatic equilibrium; all of the intermediate models between the white dwarf peak and the bottom of the neutron star peak are unstable against collapse.

The radii of the neutron star models are shown in Figure 3. Except for the lowest 


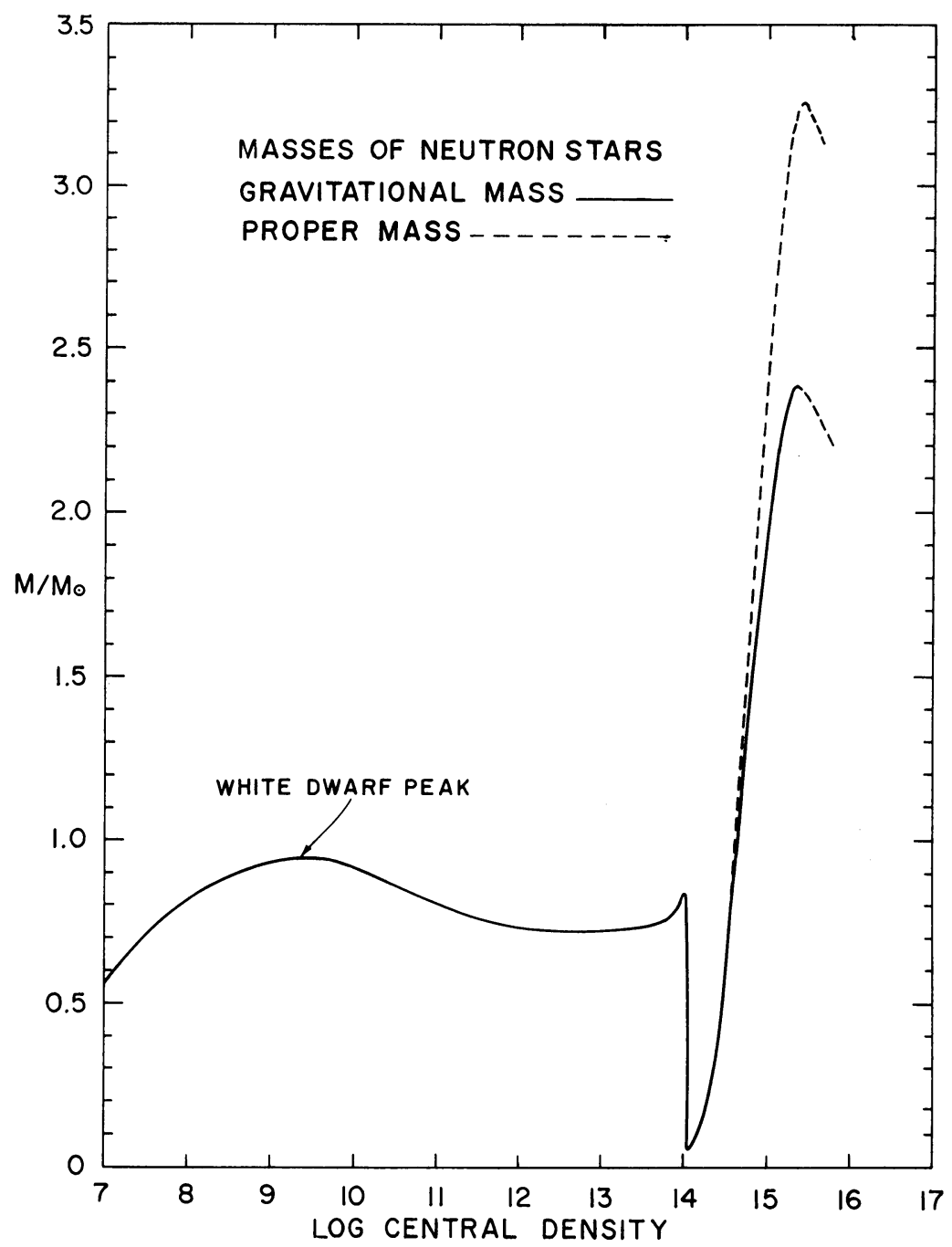

Fig. 2. Masses of neutron star models, including the white dwarf range of central densities.

mass models, these radii are remarkably insensitive to the mass, being in the vicinity of $13 \mathrm{~km}$.

The interior density distribution of a star with just over half a solar mass is shown in Figure 4. The density varies remarkably slowly over most of the interior, but there is a significant bulge at lower densities in the outermost part of the star. This bulge consists entirely of a mixture of ions and electrons, with no neutrons being present. This bulge would form the outer part of the crystalline crust.

From a model of a neutron star it is possible to calculate the moment of inertia, which is of interest for the determination of the rotational energy that the model may have for any given rotational period. It is necessary to make some small general 


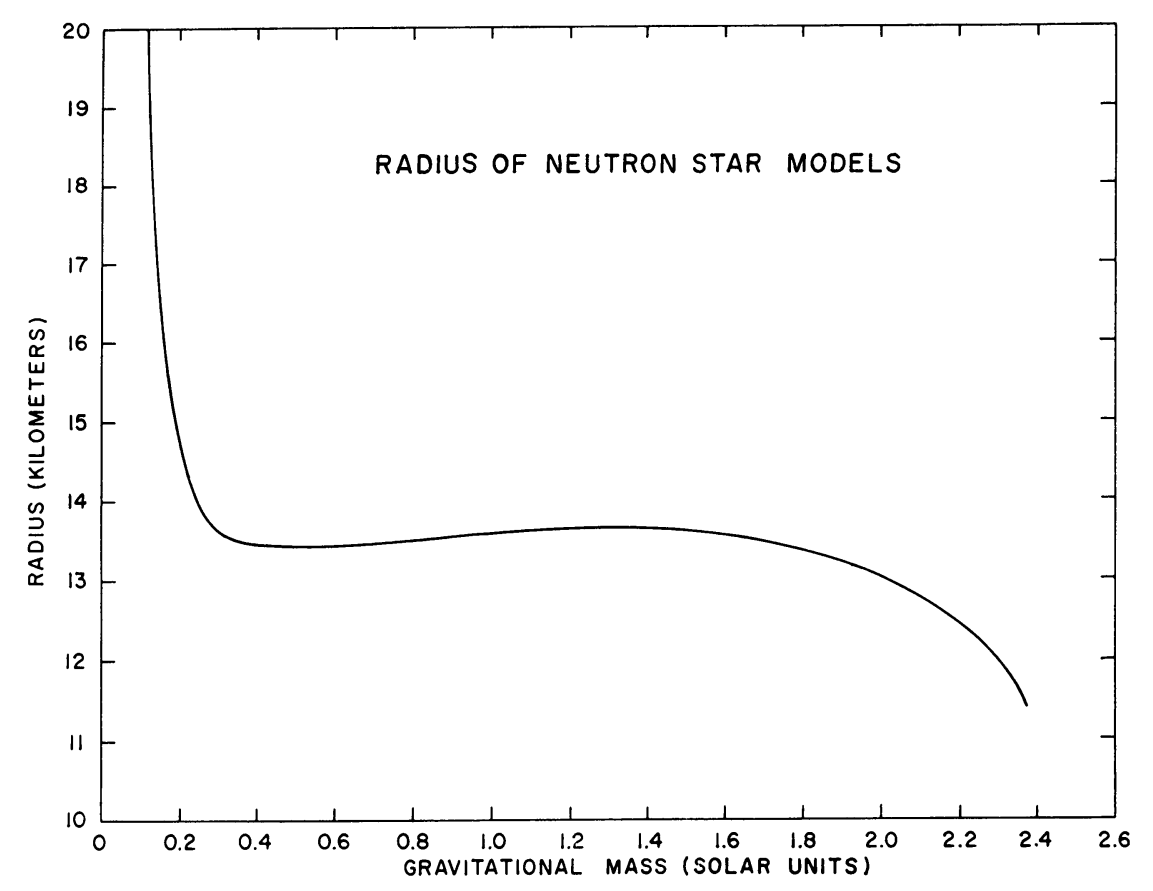

Fig. 3. Radii of the neutron star models.

relativistic corrections to the calculated moments of inertia to take into account the dragging of the inertial frames which occurs at the high gravitational potentials present in neutron star models. Then for given values of the period and the rate of change of period of a neutron star model, the rate of loss of rotational energy can be calculated. For the Crab Nebula, we know that a minimum rate of energy input to the energetic electrons in the nebula is about $10^{38} \mathrm{erg}$ per second. This must represent a minimum rate of loss of rotational energy, since additional rotational energy may be dissipated in other forms, such as the acceleration of energetic ions and the generation of internal heat. For the models shown in Figure 2, the minimum mass of neutron star which can be attributed to the Crab Nebula pulsar is about 0.4 solar masses. If the mass of the neutron star is much greater than this lower limit, close to the maximum allowable mass, then as much as a factor of 20 additional rate of energy loss would be present in the neutron star (Cohen and Cameron, 1969).

In the outer regions of the star, where ions are present, and except at the very outermost surface, the electrons are highly relativistic, and therefore their motions are only minutely perturbed by the localization of positive charges on the ions. Therefore two adjacent ions are not significantly shielded from each other by the electrons in which they are immersed, as would be the case in an ordinary plasma. Hence there is a large electrostatic repulsion between adjacent ions, which gives an interaction energy of about $1 \mathrm{MeV}$ in a typical case. For temperatures at which the 


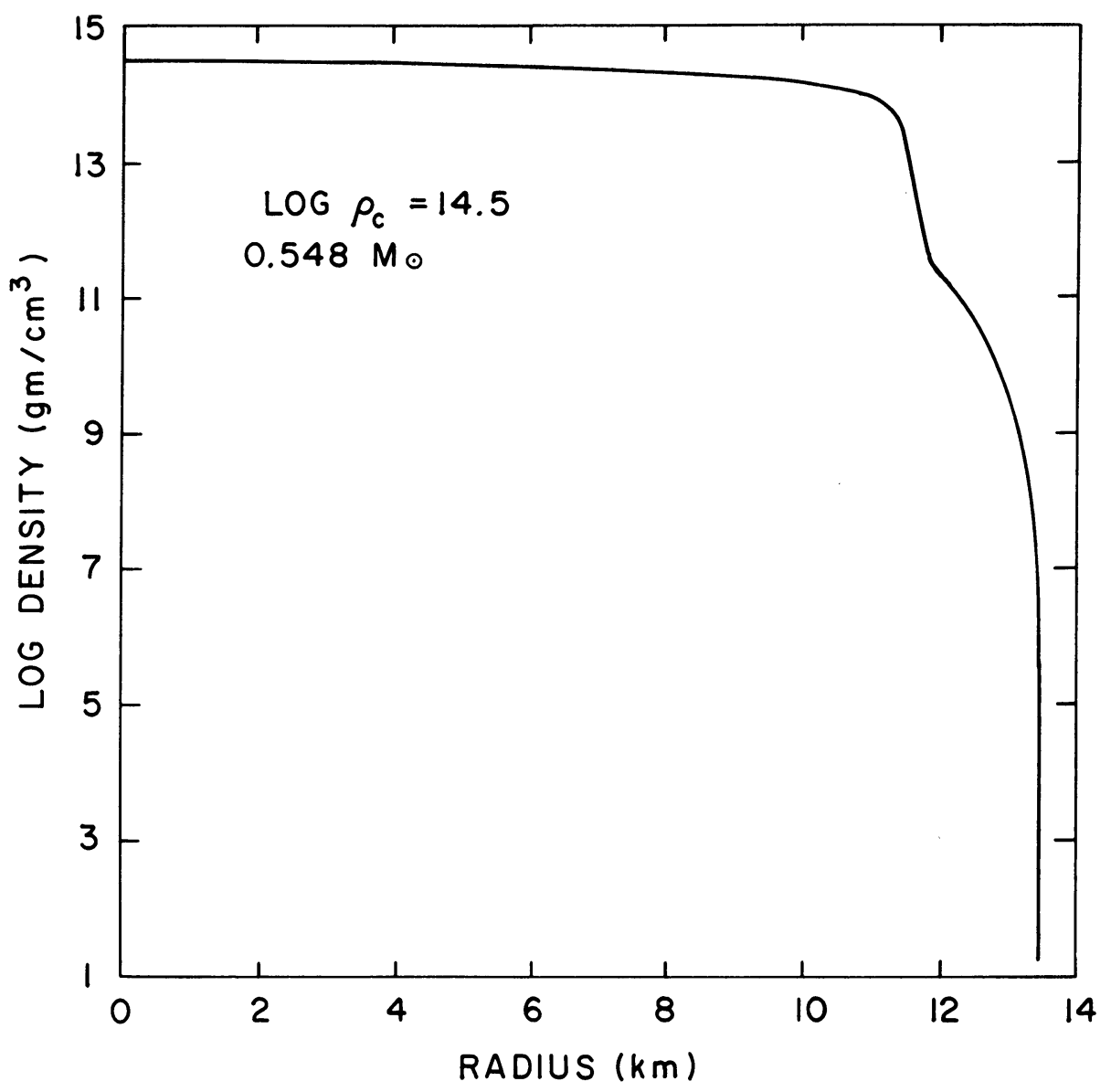

Fig. 4. Internal density distribution of a neutron star model.

typical kinetic energy of the ions is less than about one per cent of this value, the medium seeks a minimum energy configuration by freezing the ions into position in a lattice in which they are as far away from one another as possible, thus forming a crystal (Salpeter, 1961). This does not make much difference to the equation of state of the material, since most of the pressure is provided by the electrons, but it does provide the material with an enormous resistance to shear deformations (Ruderman, 1968). Toward the inner part of the ionic layer, the partial density in the form of neutrons greatly exceeds that in the form of ions, but nevertheless the crystalline lattice should still persist because of these coulomb effects.

Inside the ionic layer, the neutrons exist at less than the normal partial density of neutrons in nuclear matter, which is about $2 \times 10^{14} \mathrm{~g} / \mathrm{cm}^{2}$. In this regime of lower density, nuclear forces are predominantly attractive, and this is expected to have a profound influence upon the properties of the neutron fluid. The attractive forces 
between the neutrons at the top of the Fermi sea are expected to make them act in pairs like bosons, which then gives the fluid superfluid properties. This introduces an energy gap at the Fermi surface, of 1 to $20 \mathrm{MeV}$ in which no single particle states can exist. The magnitude of this energy gap is very large compared to the thermal energies anticipated in the neutron star interior, so the top of the Fermi sea of neutrons is not thermally rounded in the usual fashion. Only a small number of neutrons can be excited to a sufficiently high energy to populate the states lying above the energy gap (Ginzburg and Kirzhnits, 1965).

The protons are present in the medium just below the ionic layer with an abundance of about three per cent by number relative to the neutrons. The same attractive forces between protons act as between the neutrons, and it is therefore expected that the protons will also form a superfluid.

At greater than the normal nuclear density of neutrons, the nuclear forces become predominantly repulsive. Consequently, the normal superfluidity which is expected to exist at lower densities no longer exists. However, there is still one component of the nuclear force which has an attractive potential between neutrons or between protons, due to one of the $p$-state interactions, and it is likely that this attractive force will cause the neutrons to have an isotropic superfluidity, of a type which has never been observed in the laboratory (Ruderman, 1969a). In the deep core of the star, where many different types of hyperons may be present, the nature of the forces interacting between the various types of particles is not well enough known for any specific statements to be made about the expectations of superfluidity. However, it does appear that the repulsive forces are not strong enough to produce a nuclear ferromagnetism which would result from an alignment of the spins of the particles. If such an alignment were to occur, magnetic fields of the order of $10^{15} \mathrm{G}$ would be produced (Pearson and Saunier, 1969).

There are several immediate consequences which follow from the inability to have a normal thermal rounding of Fermi surfaces in the superfluid regions. One of these is the expectation that the nucleons will have a very small heat capacity; practically all of the heat capacity of the neutron star will result from a thermal rounding of the electron Fermi surface. The electrons do not form a superfluid. Another consequence is that many of the neutrino-antineutrino emission processes from the interior of the neutron star will be suppressed. These processes depend either upon the transformation of neutrons into protons and back again, or upon a scattering process in which a proton is induced to make a transformation from one cell of phase space to another. There is no available phase space for such processes except above the energy gap, so that the inhibition of such processes occurs by extremely large factors.

However, some modifications of these complete superfluid properties arise from the fact that the superfluids are rotating. A rotating superfluid is filled with vortices which have axes parallel to the axis of rotation. Each of the vortices has a very small core consisting of normal (not superfluid) material, and the particles in the vortices have a quantized rotation about each vortex axis. The whole assembly of vortices then rotates in a macroscopic fashion. 
It is expected that the charged particle components of a neutron star will rotate rigidly together. Even if there were no initial magnetic field present in the interior which would assure this, any relative motion of the charged particle systems would generate currents that would in turn build up magnetic fields in the interior sufficient to assure the charged particle corotation. However, the neutrons are not affected by the presence of a magnetic field in this way, and hence the neutron superfluid is expected to be only very weakly coupled to the charged particle systems. This coupling occurs principally through the normal material in the vortex cores; one of the more effective mechanisms for the interaction is the scattering of electrons from the neutron magnetic moments (Baym et al., 1969a). Additional friction between the neutrons and the charged particle systems arises from the interaction between the normal cores of the neutron and proton superfluids. For these reasons, as a rotating neutron star slows down, it is expected that the slowing of the neutron superfluid will lag behind the slowing of the charged particle system, so that the neutrons will be left rotating faster than the charged particle system and will be slowed down only as a result of the friction that does exist between the two systems (Baym et al., 1969b).

The magnetic field which is likely to exist in a pre-supernova star will be greatly compressed during the formation of a neutron star, and field strengths of the order of $10^{12} \mathrm{G}$ are expected to be produced. This is the magnitude of the neutron star magnetic field which is often invoked as being that required for the slowing down process in ordinary pulsars. The electrical conductivity in the interior of a neutron star is so enormously high, that the ohmic dissipation of the magnetic field would require a time long compared to the age of the Universe. Therefore the neutron star cannot exclude the magnetic field as a bulk effect. It is nevertheless expected that the superfluid protons will exhibit a form of the Meissner effect, in which superconductors exclude magnetic fields, by compressing the internal magnetic field into isolated bundles having a field strength somewhat greater than the average field strength, perhaps $10^{13}$ or $10^{14} \mathrm{G}$. The magnetic field will then interfere somewhat with the normal superfluid properties of the protons in the middle of the flux tubes, but the bulk of the protons between the flux tubes will be little affected by the presence of the field.

Furthermore, it is possible that the magnetic field may be permanently fixed in the interior of the neutron star by an electron effect which depends upon an initial field of the order of $10^{12} \mathrm{G}$ being produced in the interior. This effect arises from the rotation of the electrons in perpendicular orbits about the field lines, which gives a magnetic moment arising from the circular motions of the electrons. These orbital magnetic moments will then be aligned in the interior of the star; they reinforce the magnetic field and can maintain the field against dissipative processes. This effect has been called Landau orbital ferromagnetism (Lee et al., 1969).

There are two types of pulsar behavior which are generally attributed to the properties of the interior of a neutron star. These are the period discontinuities, resulting in a sudden decrease in the period of rotation, such as those which have been observed once each in the Vela and Crab pulsars, and also the sinusoidal phase variations of the pulse emission times which have been observed as residuals to fits 
to the pulsar rotational periods and their first two derivatives in the case of the Crab Nebula. These residuals have had a sinusoidal shape, and at first they were attributed to the possible presence of a planet near the Crab Nebula which would cause the center of mass of the pulsar to move backwards and forwards in the line of sight as the planet revolved around it with a period of about three months. However, the degeneration of this sinusoidal variation into a small amplitude stochastic variation about one cycle after the period discontinuity in the Crab Nebula pulsar is evidence against the influence of a planet in producing these variations. It is therefore very likely that the variation results from some internal behavior.

One suggestion, due to Ruderman (1969b), which has received a great deal of attention, is that the decrease of period which has occurred in the Vela and Crab pulsars has resulted from a large-scale fracture of the crystalline crust, resulting in a decrease of the moment of inertia of the star. In this picture, the neutron star was originally rotating much faster than it is at the present time, so that the crust would be formed with an appreciable equatorial bulge. As the neutron star slows down, the equatorial bulge will tend to flatten toward a sphere, but the high shear strength of the crystalline material will act to prevent this. Consequently, it would be expected that a large internal stress would be built up in the crust which would eventually exceed the breaking stress of the crystal, resulting in large-scale fracture of the crust and its sudden readjustment to a smaller equatorial radius.

One difficulty with this suggestion is that such a fracture would be expected to occur only a few times in the history of the slowing down of the pulsar. This makes it somewhat unlikely that two such events would have been seen in the two shortest period pulsars during the first two or three years of their observation. Furthermore, the coulomb crystal in the crust exists only as a result of the high pressure in the interior, which keeps the ions closely compacted into a lattice. In the absence of a magnetic field, it would then be possible for the non-crystalline material at the surface of the star to flow from the equator to the poles, thus relieving much of the internal stress by a redistribution of the mass. It remains to be shown that no anomalous resistive effects on the surface may occur which would allow this redistribution of mass to take place.

Another suggestion, put forward originally in an inapplicable form by Greenstein and myself (Greenstein and Cameron, 1969), is that an internal fluid instability could exist in the neutron star. In such a case, one would expect interior components of the neutron star to be rotating much faster than the surface; in this case the only available interior component that could be weakly enough coupled to the rest of the star would be the neutrons. If the angular velocity gradient in any region of the interior becomes sufficiently great, then the angular momentum per unit mass may decrease outwards, leading to a classical fluid instability which will result in interior convective motions and the outward transport of angular momentum, thus leading to an acceleration of the surface layers. Dr. Greenstein and I still think that a form of this instability is a possibility in the neutron star interior. If the friction between the superfluid neutron component of the star and the charged particle system has a sufficiently 
non-uniform distribution in radius, then the rotational vortices in the superfluid will become greatly stretched and highly deformed and tangled (Greenstein, 1970). This deformation may also occur if the ends of the vortices become anchored to irregularities in the coulomb crystal in the outer shell of the star. There is a tension exerted along the axes of the vortices, but this tension will be less in some portions of the neutron superfluid than it is at others, and therefore, it may be expected that variations will be produced in the angular velocity gradient in the interior. If the angular velocity gradient can become great enough so that a local outward decrease of angular momentum per unit mass is produced, then instabilities can set in which would mix outwards some of the superfluid neutron material possessing greater angular momentum per unit mass than that of the fluid which it displaces. In any case, since the neutrons are expected to be rotating faster than the charged particle systems, any sudden increased coupling between these systems will produce a sudden period decrease in the charged particle components of the star. Much too little is known about the detailed physics of the interior to allow a reasonable evaluation of this possibility at this time.

Although several suggestions have been made to account for the sinusoidal residuals of the pulsar phase, only one of the suggestions still appears to be possibly valid in view of the behavior of the residuals following the period decrease in the Crab Nebula pulsar. This is a suggestion due to Ruderman (1970) that an oscillation in radius of the rotational vortices of the neutron superfluid can occur. These vortices should exist in the form of a triangular lattice, providing that the tangling of the vortices is not too severe, and this lattice can undergo expansion and contraction, leading to increases and decreases of the rotation rate of the pulsar superimposed upon its bulk behavior. Indeed, Ruderman has estimated that the period of oscillation of the vortices should be a few months in the case of the Crab Nebula pulsar. This rotational variation becomes visible in the repetition rate of the pulsar emission only as a result of frictional coupling between the superfluid neutrons and the charged particle systems. If this is the correct explanation for the sinusoidal residuals, then we would have to guess that this oscillation mode of the interior is excited by some sort of internal nonlinear interaction, and the persistence of the variation for nearly a full cycle after the period decrease would then indicate that some considerable time is required before the internal effects of the period decrease to become propagated to the great bulk of the superfluid neutron interior. The tangled vortex superfluid instability suggested above as a possible explanation of the period decrease may possibly be reconciled with this situation, although the detailed physics is far from being worked out.

There is one other possible observation, which is very difficult, but which might be made to verify another consequence of the expected superfluid properties of a neutron star interior. The surface layers of a neutron star will have a greatly reduced opacity, compared to that of normal plasma, for the propagation of light along the direction of magnetic fields, and for the propagation of polarized light travelling perpendicular to magnetic fields with the electric vector perpendicular to the field direction. This results from the fact that the electron motions in the perpendicular direction in the 
strong magnetic fields under consideration are quantized with energy spacings large compared to thermal energies. Therefore the interaction of the electric vector of an electromagnetic wave with these electrons is strongly suppressed (Canuto, private communication). This should result in a greatly reduced temperature gradient in the outer layers of a neutron star, and hence an enhanced rate of cooling of the star. It is very difficult to make a realistic calculation of the opacity of the outer layers at very low temperatures, since the atoms will have their electronic orbits grossly distorted by the strong magnetic fields. Nevertheless, it would be expected that even the pulsar in the Crab Nebula should by now have cooled to a surface temperature of much less than $10^{6} \mathrm{~K}$.

However, the expected friction between the charged particle components in the star and the neutron superfluid must result in a portion of the lost rotational energy of the neutron star appearing as a heating of the neutron superfluid. The amount of heating will depend upon the rate at which the motion of the neutrons lags in slowing down behind that of the charged particle systems. If the rotation of the neutrons makes one lap with respect to the charged particles in a range of about $1 \mathrm{~h}$ to $1 \mathrm{sec}$, then the interior heating of the neutron superfluid should come into steady-state equilibrium with the surface radiation of the heat at a surface temperature in the range 1 to $10 \times 10^{6} \mathrm{~K}$. Normally, one would not expect to see any visible light resulting from this radiation, since the plasma frequency at neutron star photospheric densities is greater than the frequency of visible light. However, with the expected situation in which the cyclotron frequency of the electrons in the magnetic field is itself much greater than the plasma frequency of the electrons, then radiation is allowed to come out at frequencies much less than the plasma frequency along the magnetic field directions. Hence it can be expected that a very faint object with a very blue color should be visible at the position of the Crab Nebula pulsar at times between the pulsar flashes. Very soft X-rays should similarly be emitted between the Crab Nebula X-ray pulses. However, the conditions of observation of these effects in the Crab Nebula are so adverse that it may be better to look for them in association with the Vela pulsar, even though the expected surface temperature of the Vela pulsar would be somewhat less for that neutron star.

\section{Acknowledgements}

This work has been supported in part by the National Science Foundation and by the National Aeronautics and Space Administration.

\section{References}

Baym, G., Pethick, C., and Pines, D.: 1969a, Nature 224, 674.

Baym, G., Pethick, C., Pines, D., and Ruderman, M.: 1969b, Nature 224, 872.

Cameron, A. G. W.: 1970, Ann. Rev. Astron. Astrophys. 8, 179.

Cohen, J. M. and Cameron, A. G. W.: 1969, Nature 224, 566.

Cohen, J. M., Langer, W. D., Rosen, L. C., and Cameron, A. G. W.: 1970, Astrophys. Space Sci. 6, 228.

Cohen, J. M. and Cameron, A. G. W.: 1970, Astrophys. Space Sci. (in press). 
Ginzburg, V. L. and Kirzhnits, D. A.: 1965, Sov. Phys., JETP 20, 1346.

Greenstein, G. S. and Cameron, A. G. W.: 1969, Nature 222, 862.

Greenstein, G. S.: 1970, Nature 227, 791.

Langer, W. D., Rosen, L. C., Cohen, J. M., and Cameron, A. G. W.: 1969, Astrophys. Space Sci. 5, 259.

Langer, W. D. and Rosen, L. C.: 1970, Astrophys. Space Sci. 6, 217.

Lee, H. J., Canuto, V., Chiu, H. Y., and Chiuderi, C.: 1969, Phys. Rev. Letters 23, 390.

Pearson, J. M. and Saunier, G.: 1970, Phys. Rev. Letters 24, 325.

Ruderman, M.: 1968, Nature 218, 1128.

Ruderman, M.: 1969a, New York University Phys. Dept. Tech. Rept. No. 6/69.

Ruderman, M.: 1969b, Nature 223, 597.

Ruderman, M.: 1970, Nature 225, 619.

Salpeter, E. E.: 1961, Astrophys. J. 134, 669.

\section{Discussion}

R. Schwartz: The slide which Dr. Cameron showed on the mass-radius relation for neutron stars may be confusing. The increase in radius as $M$ increases does not mean that the binding energy decreases. The binding energy is not $\sim G M^{2} / R ; R$ has to do with the ordinary (i.e. non-neutron) matter in the envelope, which is a tiny fraction of the mass. In this region, the central density increases as $M$ goes up, despite the behaviour of $R$.

$W . J$. Cocke: One of the many arguments against a vibration model for pulsars was that vibrations would be quickly damped out by neutrino emission processes. Does the superfluidity phenomenon prevent this damping and thus allow vibrations to last for appreciable times?

A. G. W. Cameron: Superfluidity will certainly reduce neutrino emission associated with vibration. However if $\Sigma^{-}$particles are present, $N+N \rightleftarrows P+\Sigma^{-}$gives strong damping and may not be too much affected by superfluidity.

P. Horowitz: I'd like to make a comment on the demise of the 'starquake' theory, namely the frequency with which these jumps should be observed: If quakes are due to sudden crust readjustments in order to reduce the oblateness of the slowing star, then the mean time between quakes of the size observed in Vela is about $700 \mathrm{yr}$; for the Crab events of the magnitude of the September 1969, event should occur every 6 days. Thus it is remarkable that we were lucky enough to see the Vela jump, and even more remarkable that we see no further Crab jumps in a year of observing.

One further remark: If the Crab has a solid crust very much weaker than that necessary to produce jumps of the September event - for example, if it is full of cracks, or like gravel - then one gets very frequent small jumps, and this may be an explanation for the anomalous phase residuals reported by the radio and optical timing groups in the last two days. 